\title{
One-Particle Correlation Function in Evanescent Wave Dynamic Light Scattering
}

Maciej Lisicki, ${ }^{1}$ a) Bogdan Cichocki, ${ }^{1}$ Jan K. G. Dhont, ${ }^{2}$ and Peter R. Lang ${ }^{2}$

1) Institute of Theoretical Physics, Faculty of Physics, University of Warsaw, ul. Hoża 69, 00-681 Warsaw, Poland

2) Forschungszentrum Jülich, Institute for Complex Systems, D-52425 Jülich, Germany

(Dated: June 19, 2012)

In order to interpret measured intensity autocorrelation functions obtained in evanescent wave scattering, their initial decay rates have been analyzed recently [Phys. Rev. E 74, 021402 (2006), J. Chem. Phys. 132, 074704 (2010), J. Chem. Phys 135, 014701 (2011)]. A theoretical analysis of the longer time dependence of evanescent wave autocorrelation functions, beyond the initial decay, is still lacking. In this paper we present such an analysis for very dilute suspensions of spherical colloids. We present simulation results, a comparison to cumulant expansions, and experiments. An efficient simulation method is developed which takes advantage of the particular mathematical structure of the time-evolution equation of the probability density function of the position coordinate of the colloidal sphere. The computer simulation results are compared with analytic, first and second order cumulant expansions. The only available analytical result for the full time dependence of evanescent wave autocorrelation functions [Phys. Rev. Lett. 57, 17 (1986)], that neglects hydrodynamic interactions between the colloidal spheres and the wall, is shown to be quite inaccurate. Experimental results are presented and compared to the simulations and cumulant expansions.

a)Electronic mail: Maciej.Lisicki@fuw.edu.pl 


\section{INTRODUCTION}

The dynamics and microstructural ordering of macromolecules near interfaces is a fundamental scientific theme that has drawn much attention the last few years, and is of importance in many industrial and technological applications. Examples are coating processes or any process where particles are deposited onto surfaces $\stackrel{1}{ }$, membrane filtering, or any other process where dispersed particles migrate in porous media ${ }^{2}$ and more recently micro- $-\underline{3}$ and optofluidics ${ }^{4}$. Interfacial effects also play an important role in biological processes, like protein adsorption $\underline{\underline{5}}$, biofilm formation ${ }^{\underline{6}}$ or the collective motion of sperm cells near planar surfaces ${ }^{7}$.

In order to improve our understanding of these very complex processes, we have, as a first step, to develop techniques which allow us to study the underlying physics in detail on model systems. In the present contribution we are focusing on evanescent wave dynamic light scattering (EWDLS $)^{\frac{8.9}{9}}$ as a method to study the near wall dynamics of colloidal spheres. In EWDLS-experiments a laser beam is totally reflected off the interface between a glass wall and the sample solution, thereby creating an evanescent wave, which is used to illuminate a region close to the wall. The extent of the near-wall scattering volume is determined by the evanescent-wave penetration depth. By changing the incident angle of the laser beam with respect to the interface, the penetration depth can be tuned, so that a system can be probed on different length scales. Like for standard bulk dynamic light scattering, the scattered light intensity autocorrelation function (IACF) is measured. In case of suspensions of colloids in contact with a wall, the near-wall dynamics of the colloids is to a large extent determined by hydrodynamic interactions with the wall which are mediated via the solvent. The hydrodynamic friction forces differ for motion of a colloid along and perpendicular to the wall $\underline{10} \underline{\underline{12}}$. This anisotropy in the hydrodynamic interactions with the wall in combination with the evanescent illumination profile renders the interpretation of EWDLS experiments much more involved as compared to bulk scattering experiments. As a first step towards the interpretation of EWDLS correlation functions, Lan, Ostrowsky and Sornette ${ }^{\frac{8}{2}}$ derived an analytic expression for the EWDLS correlation function for spherical colloids and a hard wall, at very low concentrations of colloids, with the neglect of hydrodynamic interactions. The deviation of this expression from that for the bulk correlation function within their approximation is thus entirely due to direct interactions. Hydrodynamic interactions, however, have a pronounced effect on the form of the correlation function, and must therefore be accounted for. It seems 
not feasible to derive a similar analytic expression for the EWDLS correlation function when hydrodynamic interactions of the colloidal sphere with the wall are included. It is possible, though, to derive explicit expressions for the first cumulant, that is, the initial slope of the time-dependence of the EWDLS correlation function, which expressions have been verified experimentally $\stackrel{13}{\underline{14}}$. A general expression for the first cumulant for arbitrary concentrations of colloids can be derived, which has been evaluated explicitly to within a leading order virial expansion, and by simulations for higher concentrations in an attempt to interpret earlier experimental results at equally high concentrations $\frac{15}{17}$.

The first and second cumulant can be expressed in terms of the hydrodynamic mobilities of a colloidal particle, where a distinction must be made for motion perpendicular and parallel to the wall. The problem of determining friction and mobility coefficients of a spherical particle near an interface has a history dating back to works of Lorentz $\frac{18}{}$ and Faxén $\frac{19}{}$. In the 1960's, solutions of the Stokes equation, either numerical, or in bipolar coordinates, have been given for certain types of motion by O'Neill and coworkers ${ }^{20}-22$, who also investigated the lubrication regime of the solution, and by Brenner et al $\underline{\underline{10}} \underline{\underline{12}}$. In such a confined system the translational components of the mobility matrix $\boldsymbol{\mu}$ tend to zero when approaching the wall. This effect dominates in the dynamics of the system. These predictions are verified in a number of experimental studies, using particles of different sizes, and employing various techniques: optical trap microscopy $\stackrel{23}{ }$, nano-PIV $\underline{24}, 25$, dynamic light scattering in presence of two walls $\stackrel{26}{ }$, low coherence DLS $\underline{27}$, resonance enhanced DLS $\underline{28,29}$, and EWDLS in a system bounded by one or two walls ${ }^{8}, 13,14,30-32$.

In this work, we will need the values of the mobility matrix elements for a single sphere as a function of the particle-wall distance. To this end, Padé approximant representation will be used, as outlined by Cichocki and Jones $\underline{34}$ and earlier works of Perkins and Jones ${ }^{35,36}$. This is a very convenient tool, since it allows for analytic differentiation of the hydrodynamic mobilities in the whole range of distances from the wall with high precision.

The initial temporal decay of EWDLS correlation functions for colloids near a wall with hard-core interactions, as quantified by the first cumulant, has thus been addressed in some detail (although some issues remain to be resolved). Nothing has been done so far concerning the full time dependence of correlation functions, beyond the time regime that is described by the first cumulant expansion. In this paper we take the first step toward an understanding of the full time dependence of EWDLS correlation functions, 
where very dilute dispersions of spherical colloids are considered. Numerical simulation results are compared to analytical first- and second cumulant approximations, and to experiments.

This paper is organized as follows. In section [I] we present the general theoretical framework on which the analytical cumulant expansion as well as the simulations are based. In section III the cumulant expansion is discussed, and explicit expressions for the first two cumulants are derived. Numerical results for the various contributions for varying penetrations depths are given in the form of a table to enable easy and accurate evaluation of first and second cumulants. The new simulation method is presented in section IV and section $\nabla$ contains a comparison of experimental results with the predictions from simulations and the cumulant expansions.

\section{ONE-PARTICLE ELECTRIC FIELD CORRELATION FUNCTION}

In an EWDLS experiment the illumination profile is nonuniform. The evanescent wave enters the suspension (at the location of the wall), and its intensity varies with the perpendicular distance $z$ from the wall as $\exp (-\kappa z)$. Typically, the penetration depth $\kappa^{-1}$ is comparable to the size of colloidal particles. Given an ensemble of spherical colloids in the configuration $\left\{\mathbf{R}_{j}\right\}$, the instantaneous scattered electric field can be written as

$$
E \sim \sum_{j} \exp \left(-\frac{\kappa}{2} z_{j}\right) \exp \left(i \boldsymbol{q} \cdot \mathbf{R}_{j}\right)
$$

where $\boldsymbol{q}$ is the scattering vector. We assume that there are sufficiently many colloidal particles within the volume that is illuminated by the evanescent wave and from which scattered intensities are collected, that the scattered electric field is a Gaussian stochastic process with mean zero, thus fulfilling the assumptions of Wick's theorem. This allows to express the measured intensity correlation function

$$
g_{I}(t)=\langle I(t) I(t=0)\rangle=\left\langle E(t) E^{*}(t) E(t=0) E^{*}(t=0)\right\rangle,
$$

as a combination of averaged bilinear products of the scattered field strength. As the system is translationally invariant in the direction parallel to the wall, the average $\langle E(t) E(t=0)\rangle$ equals 0 . The resulting expression for the intensity correlation function in terms of the electric field correlation function is known as the Siegert relation, which reads

$$
g_{I}(t)=I^{2}(\boldsymbol{q})\left[1+|\hat{g}(t)|^{2}\right],
$$


where $I(\boldsymbol{q})$ is the average light intensity value, which is time-independent, as the stochastic process $E(\boldsymbol{q}, \kappa ; t)$ is stationary. Furthermore, the normalized electric field autocorrelation function $(\mathrm{EACF})$ is defined as

$$
\hat{g}(\boldsymbol{q}, \kappa ; t)=\frac{\left\langle E(\boldsymbol{q}, \kappa ; t) E^{*}(\boldsymbol{q}, \kappa ; 0)\right\rangle}{I(\boldsymbol{q})} .
$$

where $\langle\cdots\rangle$ denotes equilibrium ensemble averaging. From this definition, the initial value of the $\mathrm{EACF}$ is

$$
\hat{g}(\boldsymbol{q}, \kappa ; t=0)=1 .
$$

According to eq.(1), the correlation function $\hat{g}(\boldsymbol{q}, \kappa ; t)$, for the general $N$-particle case, is proportional to

$$
\hat{g}(\boldsymbol{q}, \kappa ; t) \propto \frac{1}{N} \sum_{i, j=1}^{N}\left\langle\exp \left\{-\frac{\kappa}{2}\left(z_{i}(t)+z_{j}(0)\right)\right\} \exp \left\{-i \boldsymbol{q} \cdot\left(\mathbf{R}_{i}(t)-\mathbf{R}_{j}(0)\right)\right\}\right\rangle .
$$

For $\kappa=0$, corresponding to an unbounded fluid, the thermodynamic limit $\lim _{\infty}$ of the right-hand side of the above expression is well-defined. However, for nonzero $\kappa$, the problem is more subtle and has been properly treated in Ref. 15. As it has been shown there, one should then consider a cubic box of volume $V=L_{x} L_{y} L_{z}$, touching the wall with its side with surface area $A=L_{x} L_{y}$. In the thermodynamic limit all dimensions of the box are simultaneously stretched, keeping the density of particles $n$ constant. To assure existence of the right-hand side of eq.(6), a factor $V / A$ must be added.

In a dilute system the description reduces to a one-particle problem and we can neglect the terms with $i \neq j$ in the sum above. Restricting to one-particle contributions to $\hat{g}$ and expressing the average using the conditional probability $P\left(\mathbf{R}, t \mid \mathbf{R}_{0}, 0\right)$ of a particle being in position $\mathbf{R}$ at time $t$, given its position $\mathbf{R}_{0}$ at $t=0$, we have, in thermodynamic limit

$$
\hat{g}(\boldsymbol{q}, \kappa ; t) \propto \lim _{\infty} \frac{V}{A} \int \mathrm{d} \mathbf{R} \int \mathrm{d} \mathbf{R}_{0} e^{-\frac{\kappa}{2}\left(z+z_{0}\right)} e^{-i \boldsymbol{q} \cdot\left(\mathbf{R}-\mathbf{R}_{0}\right)} P_{e q}\left(\mathbf{R}_{0}\right) P\left(\mathbf{R}, t \mid \mathbf{R}_{0}, 0\right) .
$$

The equilibrium distribution $P_{e q}(\mathbf{R}) \sim \exp (-\beta \Phi(z))$ is determined by the interaction potential $\Phi(z)$, depending only on the wall-particle distance $z$, and $\beta=1 / k_{B} T$, where $T$ is the temperature and $k_{B}$ is the Boltzmann constant.

Because of translational invariance in the $x y$-plane, in thermodynamic limit the conditional probability $P\left(\mathbf{R}, t \mid \mathbf{R}_{0}, 0\right)$ will be denoted by $P\left(\rho, z, z_{0}, t\right)$. The probability can only depend on the $z$-positions, the distance perpendicular to the wall, and the length of the horizontal displacement vector $\rho=|\boldsymbol{\rho}|$, defined by the relation

$$
\mathbf{R}-\mathbf{R}_{0}=\boldsymbol{\rho}+\left(z-z_{0}\right) \hat{\boldsymbol{e}}_{z},
$$




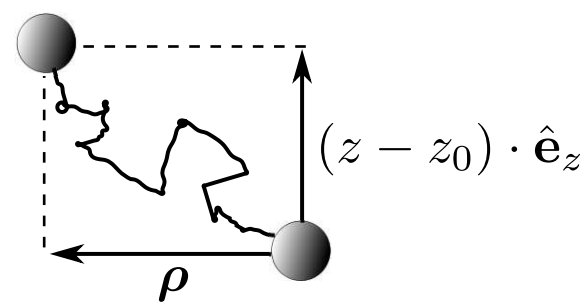

11111111111111111111111

Figure 1. Geometry of the system. The conditional probability density depends on the distance from the wall and length of the parallel displacement vector $\rho$.

and marked in Fig. 1, We similarly decompose $\boldsymbol{q}$ into components parallel and perpendicular to the wall

$$
\boldsymbol{q}=q_{\perp} \hat{\boldsymbol{e}}_{z}+\boldsymbol{q}_{\|}
$$

Next, we introduce new variables $\left(\mathbf{R}+\mathbf{R}_{0}\right) / 2$ and $\mathbf{R}-\mathbf{R}_{0}$ in eq.(7), and integrate over $\left(x+x_{0}\right) / 2$ and $\left(y+y_{0}\right) / 2$. This leads to a factor $A$.

Since the normalization factor in $P_{e q}(\mathbf{R})$ is of the order of $V$, after performing the thermodynamic limit, us the right-hand side of eq.(17) becomes

$$
\int_{a}^{\infty} \mathrm{d} z \int_{a}^{\infty} \mathrm{d} z_{0} e^{-\frac{\kappa}{2}\left(z+z_{0}\right)} e^{-i q_{\perp}\left(z-z_{0}\right)} e^{-\beta \Phi\left(z_{0}\right)} \int \mathrm{d} \boldsymbol{\rho} e^{-i \boldsymbol{q}_{\|} \cdot \boldsymbol{\rho}} P\left(\rho, z, z_{0}, t\right) .
$$

Here, the lower integration limit is the radius $a$ of the spherical colloid, which corresponds to the closest approach of the sphere to the wall. Using that the initial value of the conditional probability distribution $P\left(\mathbf{R}, t=0 \mid \mathbf{R}_{0}, 0\right)=\delta\left(\mathbf{R}-\mathbf{R}_{0}\right)$, we find the initial value of (10) as

$$
\int_{a}^{\infty} \mathrm{d} z e^{-\kappa z} e^{-\beta \Phi(z)}
$$

Using the normalization condition (5), we express the EACF as

$$
\hat{g}(\boldsymbol{q}, \kappa ; t)=\frac{\int_{a}^{\infty} \mathrm{d} z \int_{a}^{\infty} \mathrm{d} z_{0} e^{-\frac{\kappa}{2}\left(z+z_{0}\right)} e^{-i q_{\perp}\left(z-z_{0}\right)} e^{-\beta \Phi\left(z_{0}\right)} \widetilde{P}\left(q_{\|}, z, z_{0}, t\right)}{\int_{a}^{\infty} \mathrm{d} z e^{-\kappa z} e^{-\beta \Phi(z)}},
$$

where we introduced a two dimensional Fourier transform of the probability density function

$$
\widetilde{P}\left(q_{\|}, z, z_{0}, t\right)=\int \mathrm{d} \boldsymbol{\rho} e^{-i \boldsymbol{q}_{\|} \cdot \boldsymbol{\rho}} P\left(\rho, z, z_{0}, t\right)
$$


This expression will be used as a basis for the Brownian dynamics simulation in order to obtain numerical values for the EWDLS correlation function. Note that in case of a hard-core interaction potential ( $\Phi=0$ when there is no overlap and $\Phi=\infty$ otherwise), the denominator in (12) reduces to $\exp (-\kappa a) / \kappa$ where $a$ is the radius of the colloidal sphere, yielding

$$
\hat{g}(\boldsymbol{q}, \kappa ; t)=\kappa \exp (\kappa a) \int_{a}^{\infty} \mathrm{d} z \int_{a}^{\infty} \mathrm{d} z_{0} e^{-\frac{\kappa}{2}\left(z+z_{0}\right)} e^{-i q_{\perp}\left(z-z_{0}\right)} e^{-\beta \Phi\left(z_{0}\right)} \widetilde{P}\left(q_{\|}, z, z_{0}, t\right)
$$

In order to explicitly evaluate the EACF in eq.(14), the time dependence of the (partially Fourier transformed) conditional probability density function (pdf) $\widetilde{P}\left(q_{\|}, z, z_{0}, t\right)$ must be specified. For this purpose we will return to eq. (77), in which the conditional probability distribution $P\left(\mathbf{R}, t \mid \mathbf{R}_{0}, 0\right)$ satisfies the Smoluchowski equation for a single colloidal sphere in presence of a wall

$$
\frac{\partial P\left(\mathbf{R}, t \mid \mathbf{R}_{0}, 0\right)}{\partial t}=\mathcal{D} P\left(\mathbf{R}, t \mid \mathbf{R}_{0}, 0\right)
$$

Taking an arbitrary phase-space function $h(\mathbf{R})$ and again denoting the potential of interactions with the wall by $\Phi$, the Smoluchowski operator $\mathcal{D}$ is given by

$$
\mathcal{D} h(\mathbf{R})=\frac{\partial}{\partial \mathbf{R}} \cdot \mathbf{D}(z) \cdot\left[\frac{\partial}{\partial \mathbf{R}}+\beta \frac{\partial \Phi}{\partial \mathbf{R}}\right] h(\mathbf{R}) .
$$

The diffusion matrix $\mathbf{D}(z)$ is connected with the mobility matrix via the relation

$$
\mathbf{D}(z)=k_{B} T \boldsymbol{\mu}(z) .
$$

The mobility matrix $\boldsymbol{\mu}$, which relates the force acting on a spherical particle to its velocity, has the following structure in the considered frame of reference ( $z$-axis perpendicular to the wall, pointing into the fluid)

$$
\boldsymbol{\mu}(z)=\left(\begin{array}{ccc}
\mu_{\|}(z) & 0 & 0 \\
0 & \mu_{\|}(z) & 0 \\
0 & 0 & \mu_{\perp}(z)
\end{array}\right)
$$

where the elements $\mu_{\|, \perp}$ depend only on the wall-particle distance $z$.

As already discussed in the introduction, the $z$-dependencies of the scalar mobilities are well-known $\underline{10,11,20}$. For large distances from the wall $(z \rightarrow \infty)$, the mobility functions $\mu_{\|, \perp}(z)$ tend to the bulk mobility coefficient $\mu_{0}$, which is related to the bulk diffusion coefficient $D_{0}=k_{B} T \mu_{0}$. In the limit of infinite penetration depth $(\kappa \rightarrow 0)$, there are 
no effects of interaction with the wall and the expression (14) reduces to the standard exponential expression $\hat{g}(\boldsymbol{q}, \kappa ; t)=\exp \left\{-D_{0} q^{2} t\right\}$ for free diffusion.

In section IV] we discuss how the Smoluchowski equation, in combination with expression (14), will be used in a simulation scheme to obtain numerical results for the correlation function. These results will be compared to a cumulant expansion, which is discussed in the subsequent section.

\section{CUMULANT EXPANSION OF THE EACF}

Using the formal solution of the Smoluchowski equation (15)

$$
P\left(\mathbf{R}, t \mid \mathbf{R}_{0}, 0\right)=e^{\mathcal{D} t} P\left(\mathbf{R}_{0}\right)=e^{\mathcal{D} t} \delta\left(\mathbf{R}-\mathbf{R}_{0}\right),
$$

the EACF can be expanded in powers of the time evolution operator $\mathcal{D}$, which leads to the cumulant expansion. Inserting eq.(19) into the expression for the EACF leads to

$$
\hat{g}(\boldsymbol{q}, \kappa ; t) \propto \int \mathrm{d} \mathbf{R} \int \mathrm{d} \mathbf{R}_{0} e^{-\frac{\kappa}{2}\left(z+z_{0}\right)} e^{-i \boldsymbol{q} \cdot\left(\mathbf{R}-\mathbf{R}_{0}\right)} \exp \{\boldsymbol{D} t\} \delta\left(\mathbf{R}-\mathbf{R}_{0}\right) e^{-\beta \Phi\left(z_{0}\right)},
$$

It is convenient to introduce the adjoint Smoluchowski operator defined by the scalar product

$$
\int_{z>a} \mathrm{~d} \mathbf{R} f(\mathbf{R}) \mathcal{D} h(\mathbf{R})=\int_{z>a} \mathrm{~d} \mathbf{R} h(\mathbf{R}) \mathcal{D}^{\dagger} f(\mathbf{R}),
$$

where $f$ and $h$ are arbitrary phase-space functions. From eq. (16), the adjoint operator is found after partial integrations, using that the hydrodynamic mobility functions vanish on contact with the wall, and reads

$$
\mathcal{D}^{\dagger} h(\mathbf{R})=\left[\frac{\partial}{\partial \mathbf{R}}-\beta \frac{\partial \Phi}{\partial \mathbf{R}}\right] \cdot \mathbf{D}(\mathbf{R}) \cdot \frac{\partial}{\partial \mathbf{R}} h(\mathbf{R})
$$

Since $\mathcal{D}^{\dagger}$ does not act on the variable $\mathbf{R}_{0}$, we can perform the integration over $\mathbf{R}_{0}$ in eq.(20), due to the presence of the Dirac delta distribution, to obtain

$$
\hat{g}(\boldsymbol{q}, \kappa ; t) \propto \int \mathrm{d} \mathbf{R} e^{-\beta \Phi(z)} e^{i \boldsymbol{q} \cdot \mathbf{R}} e^{-\kappa z / 2} \exp \left\{\mathcal{D}^{\dagger} t\right\} e^{-i \boldsymbol{q} \cdot \mathbf{R}} e^{-\kappa z / 2}
$$

Expanding the exponential for small times, the EACF can be written as

$$
\hat{g}(\boldsymbol{q}, \kappa ; t)=1+\mu_{1} t+\frac{\mu_{2} t^{2}}{2}+\mathrm{o}\left(t^{2}\right)
$$


where two first moments are equal to

$$
\begin{aligned}
& \mu_{1}=\frac{\left\langle\varphi^{*} \mathcal{D}^{\dagger} \varphi\right\rangle}{\left\langle e^{-\kappa z}\right\rangle}, \\
& \mu_{2}=\frac{\left\langle\varphi^{*} \mathcal{D}^{\dagger} \mathcal{D}^{\dagger} \varphi\right\rangle}{\left\langle e^{-\kappa z}\right\rangle},
\end{aligned}
$$

with $\varphi \equiv e^{-i \boldsymbol{q} \cdot \mathbf{R}} e^{-\kappa z / 2}$. Re-exponentiation of eq.(24) thus leads to

$$
\hat{g}(\boldsymbol{q}, \kappa ; t)=\exp \left(-\Gamma_{1} t+\frac{1}{2} \Gamma_{2} t^{2}+\mathrm{o}\left(t^{2}\right)\right) .
$$

where the first and second cumulants $\Gamma_{1}$ and $\Gamma_{2}$ are related to the moments in eq.(26) as

$$
\begin{aligned}
& \Gamma_{1}=-\mu_{1}, \\
& \Gamma_{2}=\mu_{2}-\mu_{1}^{2} .
\end{aligned}
$$

the first moment takes the form

$$
\mu_{1}=\Gamma_{1}=\frac{\int_{a}^{\infty} \mathrm{d} z \exp \{-\kappa z\} \exp \{-\beta \Phi(z)\}\left[D_{\|}(z) q_{\|}^{2}+D_{\perp}(z)\left(q_{\perp}^{2}+\frac{\kappa^{2}}{4}\right)\right]}{\int_{a}^{\infty} \mathrm{d} z \exp \{-\kappa z\} \exp \{-\beta \Phi(z)\}} .
$$

Introducing the penetration-depth averaged diffusion coefficients for dilute suspension, defined for an arbitrary z-dependent function by

$$
\langle B\rangle_{\kappa}=\frac{\int_{a}^{\infty} \mathrm{d} z \exp \{-\kappa z\} \exp \{-\beta \Phi(z)\} B(z)}{\int_{a}^{\infty} \mathrm{d} z \exp \{-\kappa z\} \exp \{-\beta \Phi(z)\}},
$$

in correspondence with the averages defined for arbitrary concentrations in Ref. 15, we may conveniently write the first cumulant as

$$
\Gamma_{1}=q_{\|}^{2}\left\langle D_{\|}\right\rangle_{\kappa}+\left(q_{\perp}^{2}+\frac{\kappa^{2}}{4}\right)\left\langle D_{\perp}\right\rangle_{\kappa} .
$$

The second moment, needed for calculation of the second cumulant, may be then derived from eq. (26) as

$$
\begin{aligned}
\mu_{2} & =q_{\|}^{4}\left\langle D_{\|}^{2}\right\rangle_{\kappa}+\left(q_{\perp}^{2}+\frac{\kappa^{2}}{4}\right)^{2}\left\langle D_{\perp}^{2}\right\rangle_{\kappa}-2 q_{\|}^{2}\left(\frac{\kappa^{2}}{4}-q_{\perp}^{2}\right)\left\langle D_{\|} D_{\perp}\right\rangle_{\kappa} \\
& +\kappa q_{\|}^{2}\left\langle D_{\|}\left(\frac{\mathrm{d} D_{\perp}}{\mathrm{d} z}-D_{\perp} \beta \frac{\mathrm{d} \Phi}{\mathrm{d} z}\right)\right\rangle_{\kappa}-\kappa\left(q_{\perp}^{2}+\frac{\kappa^{2}}{4}\right)\left\langle D_{\perp}\left(\frac{\mathrm{d} D_{\perp}}{\mathrm{d} z}-D_{\perp} \beta \frac{\mathrm{d} \Phi}{\mathrm{d} z}\right)\right\rangle_{\kappa} \\
& +\left(q_{\perp}^{2}+\frac{\kappa^{2}}{4}\right)\left\langle\left(\frac{\mathrm{d} D_{\perp}}{\mathrm{d} z}-D_{\perp} \beta \frac{\mathrm{d} \Phi}{\mathrm{d} z}\right)^{2}\right\rangle_{\kappa} .
\end{aligned}
$$


This expression corrects the one given in an appendix in Ref. 14, which contains an erroneous sign in the fourth and fifth term on the right-hand side.

We will now restrict to hard-core interaction potential

$$
\Phi(z)=\left\{\begin{array}{cc}
0 & z>a \\
\infty & z \leq a
\end{array}\right.
$$

Evaluation of eq.(33) for a hard-core potential $\Phi(z)$ is subtle. The expression

$$
-D_{\perp}(z) \beta \frac{\mathrm{d} \Phi}{\mathrm{d} z} e^{-\beta \Phi}=D_{\perp}(z) \frac{\mathrm{d}}{\mathrm{d} z}\left(e^{-\beta \Phi}\right)
$$

within the averages, reduces then to $D_{\perp}(a) \delta(z-a)$. With the neglect of hydrodynamic interactions we have $D_{\perp}(z=a) \neq 0$, and the last term on the right-hand side of eq.(33) diverges, and therefore the second cumulant does not exist. The short-time expansion of the exact solution in this (unphysical) case, which is discussed in the Appendix, shows that the first term in the series is of order $t$, which guarantees the existence of the first cumulant, while the next term is of order $t^{3 / 2}$. Due to hydrodynamic interactions, however, $D(z) \rightarrow 0$ when $z \rightarrow a$. This assures that the right-hand side of eq.(33) exists and has a finite hard-core limit.

In this case the derivation, as we show in the Appendix, leads to the second moment in the form

$$
\begin{aligned}
\mu_{2} & =q_{\|}^{4}\left\langle D_{\|}^{2}\right\rangle_{\kappa}+\left(q_{\perp}^{4}-\frac{\kappa^{4}}{16}\right)\left\langle D_{\perp}^{2}\right\rangle_{\kappa}-2 q_{\|}^{2}\left(\frac{\kappa^{2}}{4}-q_{\perp}^{2}\right)\left\langle D_{\|} D_{\perp}\right\rangle_{\kappa} \\
& +\kappa q_{\|}^{2}\left\langle D_{\perp}^{\prime} D_{\|}\right\rangle_{\kappa}+\left(q_{\perp}^{2}+\frac{\kappa^{2}}{4}\right)\left\langle\left(D_{\perp}^{\prime}\right)^{2}\right\rangle_{\kappa}
\end{aligned}
$$

where the prime denotes a derivative with respect to $z$. In addition, the penetration-depth average (see eq.(31)) for the hard-core potential (34) reduces to

$$
\langle B\rangle_{\kappa}=\kappa \int_{a}^{\infty} \mathrm{d} z \exp \{-\kappa(z-a)\} B(z) .
$$

The Appendix contains also the proof that $\Gamma_{2}$, constructed using $\mu_{1}$ and $\mu_{2}$ as in (29), is nonnegative.

We calculated values of the coefficients appearing in both moments in Table@using the method given by Cichocki and Jones $\underline{34}$. In this method, Padé approximant representation is used for the friction matrix, which relates velocity and angular velocity to the force and torque acting on the sphere. By inversion of the friction matrix we obtain the mobility matrix ${ }^{37}$. Then, using eq. (17), we calculate $D_{\perp}(z)$ and $D_{\|}(z)$. 
For convenience, in the Table we rescaled the diffusion coefficients by the single particle bulk diffusion coefficient $D_{0}=k_{B} T / 6 \pi \eta a$, introducing

$$
\bar{D}_{\perp}=\frac{D_{\perp}}{D_{0}}, \quad \bar{D}_{\|}=\frac{D_{\|}}{D_{0}}
$$

with the temperature $T$ and $\eta$ being the solvent viscosity. Once the experimental parameters $\kappa, q_{\|}, q_{\perp}$, are known, the table allows for a fast and straightforward calculation of two approximations to the EACF (44) - by means of the first cumulant

$$
\hat{g}^{(1)}(t)=\exp \left(-\Gamma_{1} t\right)
$$

and the second cumulant approximation

$$
\hat{g}^{(2)}(t)=\exp \left(-\Gamma_{1} t+\frac{1}{2} \Gamma_{2} t^{2}\right)
$$

For hard-core interactions, the cumulant expansion (24) can be performed only up to the third order for the following reason. The diffusion coefficients are non-analytical at $z=a$, and their lubrication asymptotic behaviour can be expressed in terms of the dimensionless gap width between the surface of the sphere and the wall $\varepsilon=(z-a) / a$ as $\frac{34}{3}$

$$
\begin{gathered}
\bar{D}_{\perp} \sim \varepsilon+\frac{1}{5} \varepsilon^{2} \log \varepsilon \\
\bar{D}_{\|} \sim-2(\log \varepsilon)^{-1} .
\end{gathered}
$$

The adjoint Smoluchowski operator $\mathcal{D}^{\dagger}$ contains two derivatives with respect to $z$, so that consecutive terms in the cumulant expansion contain averages of higher order derivatives of $D_{\perp}$ and $D_{\|}$. Careful analysis of the integrals in these averages, taking into account the nonanalytic behaviour of the diffusion coefficients in the proximity the wall, given by (41) and (42), leads to a conclusion that the third cumulant exists, while the fourth cumulant and all higher ones do not exist.

It is worth noticing that when the penetration depth is small (which corresponds to large values of $\kappa a$ ), the penetration-depth averages of the diffusion coefficients in Table [ become small as well. The reason for such behaviour is that in this case only the particles that are very close to the wall contribute to the average. Moreover, for those particles, their diffusion coefficients decrease due to the hydrodynamic interactions with the wall (see eqns. (41) and (42)). 
Table I. Components of first and second cumulant of the EACF for hard-core interaction between the wall and the particle. The values of the cumulants can be reproduced from eq. (32) and (36) by using the above averaged values of the diffusion coefficients. The prime denotes derivative with respect to $z$, and the bar denotes the diffusion coefficients rescaled by the single-particle bulk diffusion coefficient $D_{0}, \bar{D}_{\perp, \|}=D_{\perp, \|} / D_{0}$.

\begin{tabular}{|c|c|c|c|c|c|c|c|}
\hline \multirow[t]{2}{*}{$\kappa a$} & \multicolumn{2}{|c|}{ First cumulant } & \multicolumn{5}{|c|}{ Second cumulant } \\
\hline & $\left\langle\bar{D}_{\|}\right\rangle_{\kappa}$ & $\left\langle\bar{D}_{\perp}\right\rangle_{\kappa}$ & $\left\langle\bar{D}_{\|}^{2}\right\rangle_{\kappa}$ & $\left\langle\bar{D}_{\perp}^{2}\right\rangle_{\kappa}$ & $\left\langle\bar{D}_{\|} \bar{D}_{\perp}\right\rangle_{\kappa}$ & $a\left\langle\bar{D}_{\perp}^{\prime} \bar{D}_{\|}\right\rangle_{\kappa}$ & $a^{2}\left\langle\left(\bar{D}_{\perp}^{\prime}\right)^{2}\right\rangle_{\kappa}$ \\
\hline 0.1 & 0.884 & 0.781 & 0.796 & 0.652 & 0.715 & 0.053 & 0.029 \\
\hline 0.2 & 0.831 & 0.682 & 0.708 & 0.516 & 0.597 & 0.088 & 0.055 \\
\hline 0.3 & 0.791 & 0.612 & 0.647 & 0.429 & 0.517 & 0.115 & 0.079 \\
\hline 0.4 & 0.761 & 0.559 & 0.600 & 0.367 & 0.459 & 0.137 & 0.101 \\
\hline 0.5 & 0.736 & 0.516 & 0.563 & 0.321 & 0.414 & 0.155 & 0.121 \\
\hline 0.6 & 0.715 & 0.481 & 0.533 & 0.284 & 0.377 & 0.171 & 0.141 \\
\hline 0.7 & 0.697 & 0.451 & 0.507 & 0.254 & 0.347 & 0.184 & 0.159 \\
\hline 0.8 & 0.681 & 0.425 & 0.485 & 0.230 & 0.322 & 0.195 & 0.176 \\
\hline 0.9 & 0.667 & 0.403 & 0.466 & 0.209 & 0.300 & 0.206 & 0.192 \\
\hline 1 & 0.654 & 0.383 & 0.449 & 0.191 & 0.281 & 0.214 & 0.208 \\
\hline 1.1 & 0.643 & 0.365 & 0.434 & 0.176 & 0.264 & 0.222 & 0.222 \\
\hline 1.2 & 0.632 & 0.349 & 0.420 & 0.163 & 0.249 & 0.230 & 0.236 \\
\hline 1.3 & 0.622 & 0.335 & 0.408 & 0.151 & 0.236 & 0.236 & 0.249 \\
\hline 1.4 & 0.614 & 0.322 & 0.396 & 0.141 & 0.224 & 0.242 & 0.262 \\
\hline 1.5 & 0.605 & 0.310 & 0.386 & 0.131 & 0.213 & 0.247 & 0.274 \\
\hline 1.7 & 0.591 & 0.288 & 0.368 & 0.116 & 0.194 & 0.256 & 0.297 \\
\hline 1.9 & 0.578 & 0.270 & 0.352 & 0.103 & 0.179 & 0.264 & 0.318 \\
\hline 2 & 0.572 & 0.262 & 0.345 & 0.097 & 0.172 & 0.268 & 0.328 \\
\hline 5 & 0.473 & 0.140 & 0.236 & 0.031 & 0.078 & 0.311 & 0.518 \\
\hline 7 & 0.442 & 0.108 & 0.206 & 0.019 & 0.056 & 0.317 & 0.587 \\
\hline 9 & 0.420 & 0.088 & 0.186 & 0.013 & 0.043 & 0.319 & 0.636 \\
\hline
\end{tabular}




\section{SIMULATION PROCEDURE}

A fast numerical scheme for the calculation of the EACF for all times relies on the structure of the hydrodynamic mobility matrix $\boldsymbol{\mu}$. Because of translational invariance in the $x y$-plane parallel to the wall, the structure of the probability distribution function (pdf) in that plane is purely Gaussian, with a $z$-dependent diffusion coefficient $D_{\|}(z)$. This leads to the idea that the pdf for the Brownian particle can be recovered by tracing only the trajectory in the $z$-direction, where the diffusion coefficient is given by $D_{\perp}(z)$, and account for the Gaussian evolution in the parallel plane in a different way. We start from the Smoluchowski equation (15), written in a more explicit form as

$$
\frac{\partial P\left(\rho, z, z_{0}, t\right)}{\partial t}=\frac{\partial}{\partial z}\left(D_{\perp}(z) \frac{\partial P\left(\rho, z, z_{0}, t\right)}{\partial z}\right)+D_{\|}(z)\left(\frac{\partial^{2}}{\partial x^{2}}+\frac{\partial^{2}}{\partial y^{2}}\right) P\left(\rho, z, z_{0}, t\right) .
$$

Because of symmetry, the pdf $P$ depends only on the initial and final vertical coordinates $z$ and $z_{0}$, and displacement in the $x y$-plane parallel to the wall, represented by the vector $\boldsymbol{\rho}$ (see Fig,1).

Performing the Fourier transformation in the $x y$-plane, and denoting the transformed pdf by $\widetilde{P} \equiv \widetilde{P}\left(q_{\|}, z, z_{0}, t\right)$, as in eq.(13) $)$, we obtain an equation of the form

$$
\frac{\partial \widetilde{P}}{\partial t}=\frac{\partial}{\partial z}\left(D_{\perp}(z) \frac{\partial \widetilde{P}}{\partial z}\right)-q_{\|} D_{\|}(z) \widetilde{P}
$$

This can be regarded as a one-dimensional diffusion-reaction equation, or a diffusion equation with a probability sink. The reduction from the three-dimensional Smoluchowski equation to one dimension allows for significant acceleration of the simulation.

Putting eq.(44) in the following form

$$
\widetilde{P}\left(q_{\|}, z, z_{0}, t\right)=e^{\mathcal{L} t} \widetilde{P}\left(q_{\|}, z, z_{0}, t=0\right),
$$

we can decompose the evolution operator $\mathcal{L}$ into two distinct parts: the diffusion operator $\mathcal{L}_{1}=\partial_{z}\left[D_{\perp}(z) \partial_{z}(\cdot)\right]$, and the probability-sink operator $\mathcal{L}_{2}=-q_{\|}^{2} D_{\|}(z)$. To obtain a suitable numerical scheme, we use the Trotter identity $\underline{33}$ and treat each part in a different way. The evolution according to $\mathcal{L}_{1}$ can be obtained from a Brownian dynamics numerical scheme. We generate trajectories of Brownian particles in the $z$-direction. Obtained trajectories build the corresponding probability distribution and are then used as an ensemble for calculating averages. Every Brownian dynamics step is followed by application of $\mathcal{L}_{2}$. We account for this type of evolution by introducing a decay of $\widetilde{P}$, 
according to

$$
\widetilde{P}\left(q_{\|}, z, z_{0}, t+\Delta t\right)=e^{\mathcal{L}_{2} \Delta t} \widetilde{P}\left(q_{\|}, z, z_{0}, t\right)=\widetilde{P}\left(q_{\|}, z, z_{0}, t\right) e^{-q_{\|}^{2} D_{\|}(z) \Delta t}
$$

The weight a particle contributes to the probability distribution is dependent on its trajectory. For evaluation of the diffusion coefficients $D_{\|}(z)$ and $D_{\perp}(z)$, we use the results of Cichocki and Jones $\underline{34}$.

Results are presented in terms of dimensionless quantities, where lengths are scaled to the particle radius $a$, while the timescale is set by the structural relaxation time $\tau_{D}=a^{2} / D_{0}$, i.e. the time needed for the particle to diffuse over its own radius. For comparison with experiments, $D_{0}$ is calculated for given experimental conditions and the hydrodynamic radius of the particles is determined from bulk measurements.

To generate the trajectories of Brownian particles, we employ the first-order ErmakMcCammon scheme $e^{38}$, modified by the presence of the sink term. Particle positions in the $z$-direction are updated in each time step according to

$$
z(t+\Delta t)=z(t)+D_{\perp}^{\prime}(z) \Delta t+\sqrt{2 D_{\perp}(z) \Delta t} n
$$

where $n$ is a Gaussian random variable with zero mean and unit variance. Next, we account for the probability-sink term, according to eq.(46). This procedure is repeated in every step of the simulation. The reduction of a full three-dimensional diffusion problem to a one-dimensional diffusion-reaction equation allows for fast and efficient calculation of the EACF. To obtain the correlation functions, $N=10^{5}$ particle trajectories have been generated. For typical values of the parameters, the relative error is of order of $0.3 \%$ at $t=\tau_{D}$ and of order $0.8 \%$ at $t=3 \tau_{D}$. Therefore the error bars are smaller than the size of points and were not marked in the graphs.

Hydrodynamic interactions are of essential importance to describe the dynamics of spherical colloids near walls. This can be seen from Fig, 2 , where a comparison of EACF's is made as obtained from our simulations, which account for hydrodynamic interactions, and the analytical solution ${ }^{8}$ (which we will call LOS for Lan-Ostrowsky-Sornette), where hydrodynamic interactions are completely neglected. The figures $2 \mathrm{a}$ and $\mathrm{b}$ are for two different penetration depths. The data points are the simulation results and the lowest curve are the results for a constant diffusion coefficient where hydrodynamic interactions are neglected. As can be seen, there is a large deviation between the results with and without hydrodynamic interactions, also for the initial slope. 

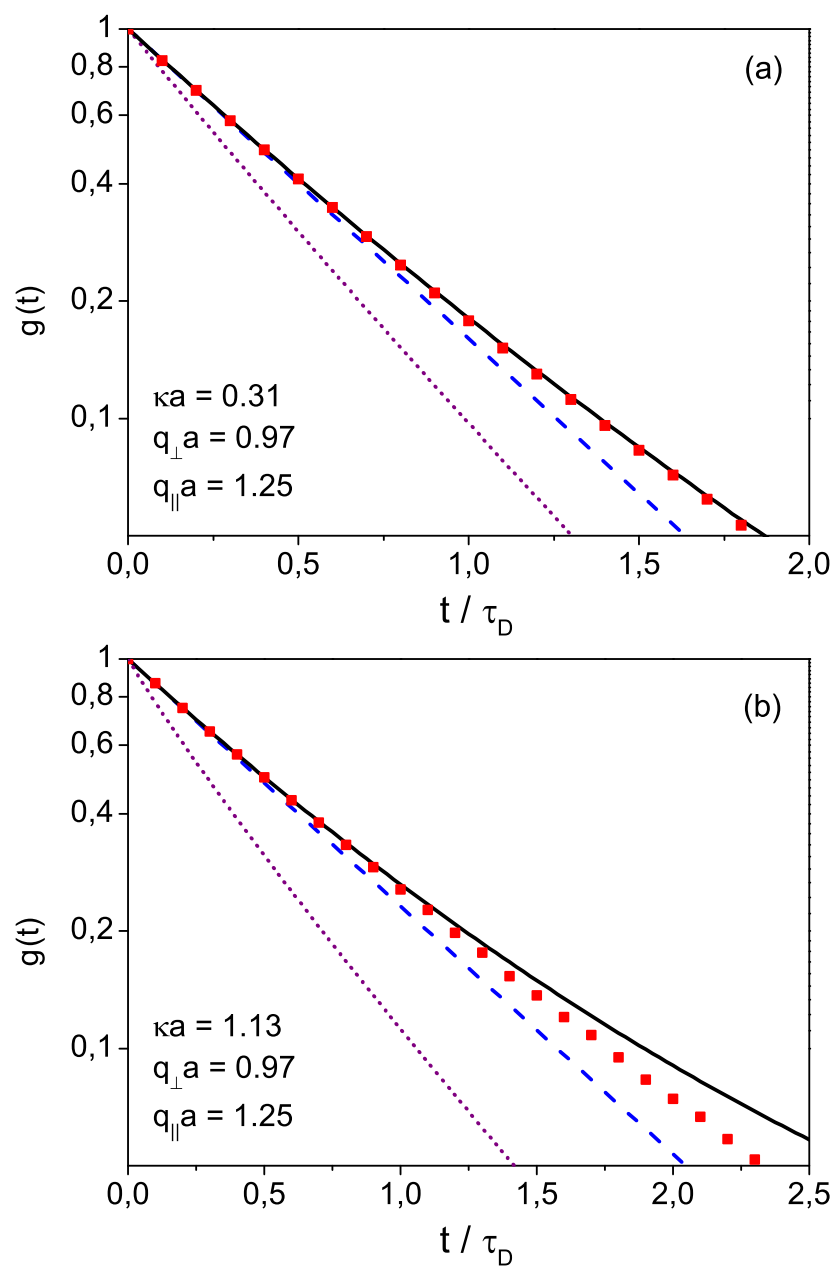

Figure 2. Numerical values for the EACF for two penetration depths at fixed values of the wave-vector components parallel and perpendicular to the wall, typical for experiments. The data points are simulation results. The dotted curve is the EACF obtained by neglecting hydrodynamic interactions (LOS solution). The dashed curve is an approximation by means of the first cumulant, while the solid line is the second cumulant approximation, given by eqns.(39) and (40), respectively.

Also included in Fig. 2 are the results from the first and second order cumulant approximation. In table Ine accuracies of the first- and second cumulant approximations (given by eqns. (39) and (40), respectively) are quantified as a function of the penetration depth. The two right columns give the values of the EACF at which the first- and second cumulant expansion deviates by $5 \%$ from its numerically calculated value, respectively. For smaller values of the EACF the deviations are larger than 5\%. Apparently, the second cumulant approximation is almost exact for sufficiently large penetration depths, typically of order $\kappa a \sim 1$, and becomes less accurate for small penetration depth (large 
Table II. Values of the EACF $\hat{g}$, for varying penetration depth (left column), at which the deviation of the first cumulant approximation $\hat{g}_{1}^{(1)}$ (middle column) and two-cumulant approximation $\hat{g}_{1}^{(2)}$ (right column) from the simulation results are $5 \%$. The values of the scattering vector components are $q_{\|} a=q_{\perp} a=1$.

\begin{tabular}{cll}
\hline \hline$\kappa a$ & $\hat{g}_{1}^{(1)}$ & $\hat{g}_{1}^{(2)}$ \\
\hline 0.3 & 0.36 & 0.04 \\
0.5 & 0.40 & 0.14 \\
0.7 & 0.43 & 0.22 \\
1 & 0.45 & 0.3 \\
1.2 & 0.46 & 0.31 \\
1.5 & 0.47 & 0.36 \\
\hline \hline
\end{tabular}

$\kappa a)$. Then the cumulant values calculated for large $\kappa a$ with the help of Table \ can be used to predict the initial decay rate of the EACF.

\section{EXPERIMENTAL}

The EWDLS experiments were performed on poly (methyl methacrylate) (PMMA) particles, which were sterically stabilized by a thin poly-12-hydrohystearic acid layer (purchased from Andrew Schoffield, University of Edinburgh), in a refracting index matching solvent mixture $\left(n_{2}=1.498\right)$ consisting of cis-decaline and tetraline $(20 / 80 \mathrm{w} / \mathrm{w})$. The solvent was chosen to minimize suspension turbidity and thereby multiple scattering, as well as van der Waals interactions. The particle concentration was determined by drying a small aliquot of the suspension and weighing the remaining polymer. We employed standard DLS measurements to determine the particles hydrodynamic radius, where we used three different methods to analyze the IACF namely cumulant analysis, stretched exponential fitting and inverse Laplace transformation. The three methods gave a hydrodynamic radius of $R_{H}=98 \mathrm{~nm}$ varying less than $1 \mathrm{~nm}$ and indicated a size polydisperisty of less than five percent. The latter finding is confirmed by the observation that the suspensions crystallize at sufficiently large particle volume fractions, which is usually regarded as indication, that polydispersity effects can be excluded. As the particles are not charged, and suspended in a non-polar solvent with matching dielectric properties, DLVO interactions between the particles as well as between the wall and the particles can 
be neglected to a good approximation and the system may be regarded as a suspension of hard spheres.

EWDLS experiments were performed with a home built instrument, based on a triple axis diffractometer, which has been described in detail elsewhere $\frac{14}{4}$. As a light source we used a frequency doubled Nd/Yag Laser (Excelsior; Spectra Physics) with a vacuum wavelength of $\lambda=532 \mathrm{~nm}$ and a nominal power output of $150 \mathrm{~mW}$. In a typical EWDLS experiment, a laser beam is incident on the interface between the sample and the glass wall at an angle $\alpha_{i}$ greater than the critical angle, resulting in total reflection which is accompanied by an evanescent wave within the sample. The wave penetrates the medium, and the penetration depth can be expressed as

$$
\frac{2}{\kappa}=\lambda / 2 \pi \sqrt{\left(n_{1} \sin \alpha_{i}\right)^{2}-n_{2}^{2}},
$$

where $\lambda$ is the laser vacuum wavelength, $n_{1}$ and $n_{2}$ are the refractive indices of glass and solvent, respectively. The angle of incidence is as in Snell's law, i. e. the angle between the interface normal and the incident beam. The scattering vector $\boldsymbol{q}=\boldsymbol{k}_{s}-\boldsymbol{k}_{e}$ is the wave vector difference between the scattered wave and the evanescent wave. The components $q_{\|}$and $q_{\perp}$ parallel and perpendicular to the wall, respectively, can be varied independently through variation of the angles $\alpha_{r}$ and $\theta$, where $\alpha_{r}$ is the angle between the unit vector along $\boldsymbol{k}_{s}$, and the wall, while $\theta$ is the angle between the projection of $\boldsymbol{k}_{s}$ onto the interface and $\boldsymbol{k}_{e}$. The two scattering-vector components are related to these two angles as

$$
\begin{aligned}
q_{\|} & =2 \pi n_{2} \sqrt{1+\cos ^{2} \alpha_{r}-2 \cos \alpha_{r} \cos \theta} / \lambda, \\
q_{\perp} & =2 \pi n_{2} \sin \alpha_{r} / \lambda .
\end{aligned}
$$

The time dependence of the correlation function varies with the penetration depth and the two components of the scattering vector. For smaller penetration depths the nearwall dynamics is probed, while for large penetration depths the form of the correlation function will resemble that of the bulk correlation function. For relatively large $q_{\|}$the diffusive dynamics along the wall is primarily probed, while for large $q_{\perp}$ the diffusive motion from and away from the wall is probed.

It turns out that there are huge fluctuations in the scattered intensity when the volume fraction of colloids is less than about 0.1. This is probably due to a too small number of colloids within the scattering volume. These huge fluctuations render accurate measurements of the IACF long time part at very low concentrations not feasible. We 


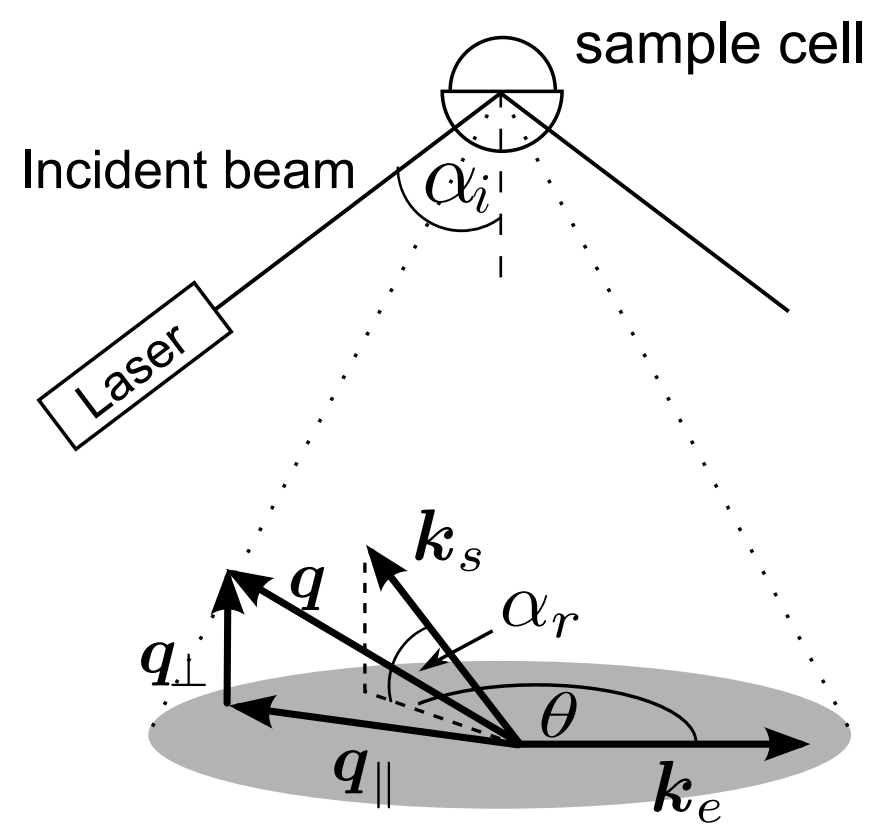

Figure 3. Sketch of an EWDLS setup. The sample cell is illuminated by the evanescent wave of a totally internally reflected beam. The geometry of beams determines the scattering vector $\boldsymbol{q}$ and the penetration depth $2 / \kappa$. The magnitudes of the incident and scattered wave vectors are equal $\left|\boldsymbol{k}_{e}\right|=\left|\boldsymbol{k}_{s}\right|$.

therefore have to restrict the experiments to a volume fraction of 0.1. At this concentration the effects of inter-colloidal interactions can not be fully neglected, so that only a semi-quantitative comparison to the theory is feasible.

Experimental results are given in Fig, 4, the empty triangles, for a concentration corresponding to a volume fraction of 0.106 . The left panel of plots if for a small penetration depth $(\kappa a=1.3)$, and the right panel is for a large penetration depth $(\kappa a=0.77)$. From top to bottom, the parallel component of the scattered wave vector increases (from $q_{\|} a=1.06,1.49$, to 1.92). For all plots, the perpendicular component of the scattered wave vector is fixed to $q_{\perp} a=1.49$. The filled squares are the simulation results, the solid lines correspond to the second cumulant approximation, and the dotted lines to the LOS predictions, where hydrodynamic interactions are neglected. As can be seen, the simulation results for infinite dilution are in reasonable agreement with the experiments. There are deviations at later times, which can have two reasons. First of all the concentration is not low enough to be able to completely neglect inter-colloidal interactions. Bulk DLS measurements actually show that in the relevant q-range the IACF decay rates measured at $\phi=0.106$ are about $30 \%$ smaller than those obtained at infinite dilution. Secondly, 

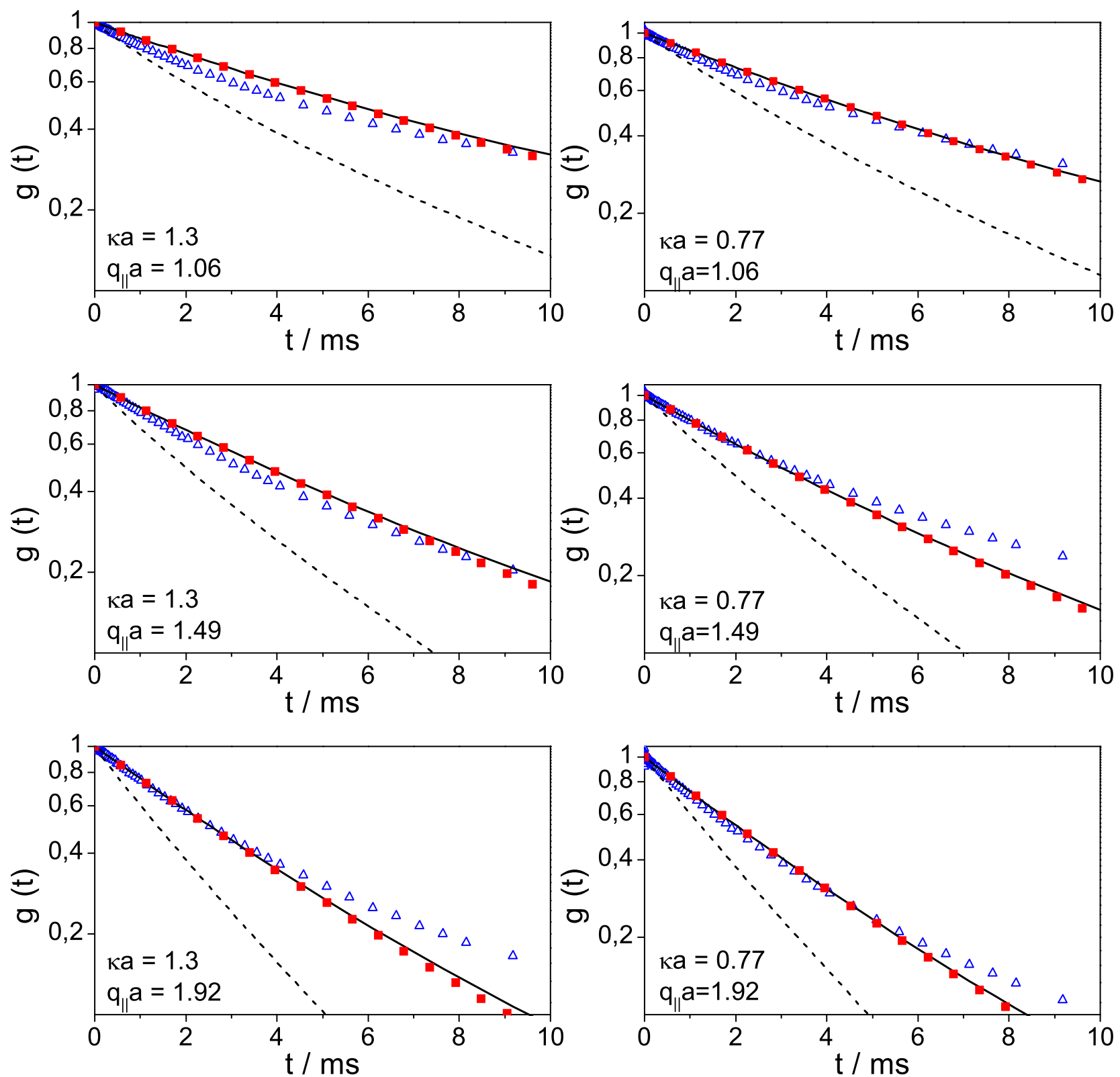

Figure 4. Experimental correlation functions at a volume fraction of 0.106 , for two penetration depths $\kappa=1.30 / a$ and $0.77 / a$. Here $q_{\perp}=1.49 / a$ is the same for all experiments while $q_{\|}$ varies from $1.06 / a$ to $1.92 / a$, as indicated in the figures. The square symbols refer to simulation predictions, while the empty triangles are the experimental results. The solid lines refer to the second cumulant approximation, and the dashed lines are the LOS predictions where hydrodynamic interactions are neglected. 


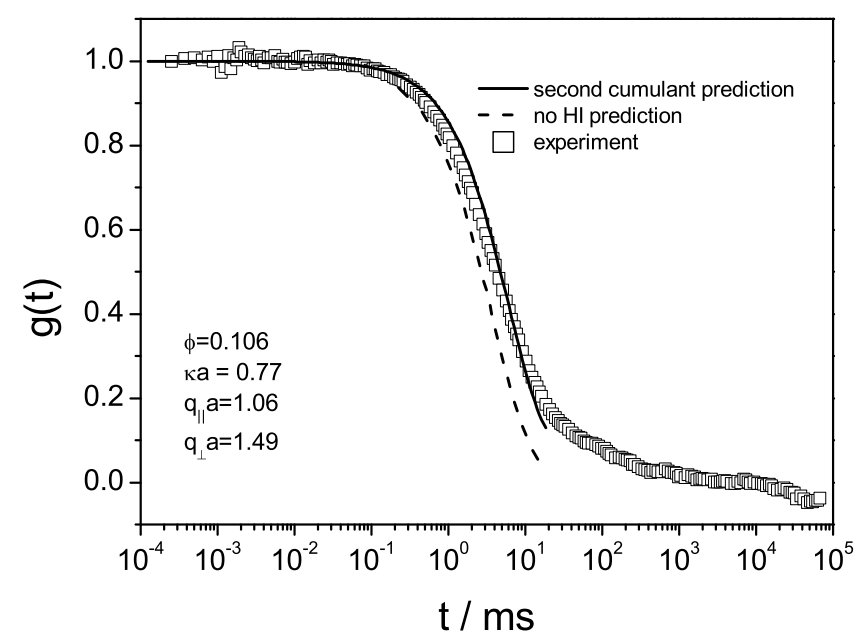

Figure 5. Experimental EACF showing a long time decay as described in the text (symbols) together with the second cumulant (full line) and the LOS (dashed line) predictions.

in many EWDLS experiments a long time decay of the IACF, as shown in Fig. 5, is observed, which so far is not yet well understood. A possible reason is $\underline{40}$, unavoidable heterodyne stray-light originating from surface defects which is scattered by colloids in the bulk of the suspension into the detector. Due to the scattering optics, only the light scattered from particles which are located in the solid angle defined by the position of the defect and the detector aperture will be detected. This corresponds to scattering vectors in the range smaller than approximately $10^{-4} \mathrm{~nm}^{-1}$ for the given set up. Taking into account the particles bulk diffusion constant this will lead to relaxation times around 10 seconds and above. This might partly explain the slower decay of the experimental data as compared to the simulations at longer times. In view of the good agreement between simulation data and the second cumulant expansion, as discussed in section [V] it is no surprise that the second cumulant approximation also describes the experimental data quite accurately. Again, the correlation functions without hydrodynamics (the dotted lines) are far off from both experiments and simulations.

\section{CONCLUSIONS}

Due to the complicated hydrodynamic interactions of colloids and a wall, it is not possible to derive a closed analytical expression for evanescent wave dynamic light scattering (EWDLS) correlation functions. At infinite dilution, where inter-colloidal interactions can be neglected, the electric field auto correlation function (EACF) for spherical colloids 
in bulk is simply equal to $\exp \left\{-q^{2} D_{0} t\right\}$, where $q$ is the scattering wave vector and $D_{0}$ is the Einstein diffusion coefficient. A similar expression for the full time dependence of the EWDLS-correlation, also at infinite dilution, has so far not been reported. It seems that the full time dependence of EWDLS-correlation functions, even for very dilute suspensions of spherical colloids, requires cumulant approximations and/or simulations. We made a comparison between first- and first-two-cumulant approximations and results obtained from a new simulation algorithm (with numerical errors that do not exceed the size of points). These results are also compared to experiments on hard-sphere like colloids, where the penetration depth and scattering wave vectors are varied. The lowest volume fraction at which reliable experimental results for the full time dependence of EWDLS-correlation functions can be obtained is about $10 \%$. The first-two-cumulant approximation is surprisingly accurate, especially for somewhat larger penetration depths. We quantified the accuracy of cumulant approximations as a function of the penetration depth in table II. This is important, since one can rely on an analytic form for the correlation function based on the known forms of the first two cumulants. Furthermore, there is reasonable agreement between the simulations and experiments. There are some deviations at long times, which can be due to inter-colloidal interactions and/or to heterodyne stray light that is scattered in forward direction by colloids residing in the bulk. For future experiments it would be desirable to use sample cells where the path length of stray light through the bulk of the sample is minimized.

Now we have an understanding of the EWDLS correlation function for very dilute suspensions of spherical colloids, the next step would be to include colloid-colloid interactions, and to extend the present approach to non-spherical colloids. This is work in progress.

\section{ACKNOWLEDGMENTS}

M.L. wishes to thank P. Szymczak for advice and providing computer time for simulations, and acknowledges support by the Foundation for Polish Science International Ph.D. Projects Programme co-financed by the EU-European Regional Development Fund. B.C. acknowledges financial support by the Deutsche Forschungsgemeinschaft Grant No. SFB-TR6 Physics of Colloidal Dispersions in External Fields, Project A1.

Experiments were performed with financial support from the EU through FP7Infrastructures ESMI (Grant agreement Number 262348). 


\section{Appendix A: Calculation of the second cumulant}

Using the definition of the adjoint Smoluchowski operator (21) and the property

$$
\mathcal{D} P^{e q}(\ldots)=P^{e q} \mathcal{D}^{\dagger}(\ldots)
$$

where $P^{e q} \sim e^{-\beta \Phi}$ is the equilibrium probability distribution, we can write the average in the second moment (26) in a very convenient form

$$
\mu_{2}=\frac{\left\langle\left|\mathcal{D}^{\dagger} \varphi\right|^{2}\right\rangle}{\left\langle e^{-\kappa z}\right\rangle} .
$$

We will now restrict ourselves to the hard-core interaction potential, but generalization to arbitrary potential is straightforward. Denoting by $\alpha=\frac{\kappa}{2}+i q_{\perp}$, and for $\varphi$ defined as under eq. (26), we have

$$
\mathcal{D}^{\dagger} \varphi=\varphi C
$$

where

$$
C=\left(-\alpha D_{\perp}^{\prime}+\alpha^{2} D_{\perp}-q_{\|}^{2} D_{\|}\right)
$$

For the second moment we get then an expression in the form

$$
\mu_{2}=\frac{\left\langle e^{-\kappa z}|C|^{2}\right\rangle}{\left\langle e^{-\kappa z}\right\rangle}=\left\langle|C|^{2}\right\rangle_{\kappa} \geq 0 .
$$

Clearly, $\mu_{2}$ is nonnegative. By direct substitution of $C$ from eq. (A4) into the above equation and using the fact that by partial integration it follows that

$$
\left\langle D_{\perp}^{\prime} D_{\perp}\right\rangle_{\kappa}=\frac{1}{2}\left\langle\left(D_{\perp}^{2}\right)^{\prime}\right\rangle_{\kappa}=\frac{\kappa}{2}\left\langle D_{\perp}^{2}\right\rangle_{\kappa}
$$

we arrive at an expression for the second moment (36). The coefficients accompanying the combinations of powers of $\kappa$ and $q_{\|, \perp}$ have been tabulated to provide a convenient tool for approximating the EACF (see Table I).

We will now show that the second cumulant $\Gamma_{2}$ is positive in this case. Consider the following nonnegative expression

$$
(\star)=\left\langle\left|-\alpha\left(D_{\perp}^{\prime}-\left\langle D_{\perp}^{\prime}\right\rangle_{\kappa}\right)+\alpha^{2}\left(D_{\perp}-\left\langle D_{\perp}\right\rangle_{\kappa}\right)-q_{\|}^{2}\left(D_{\|}-\left\langle D_{\|}\right\rangle_{\kappa}\right)\right|^{2}\right\rangle_{\kappa} \geq 0 .
$$

Again, by partial integration it can be shown that $\left\langle D_{\perp}^{\prime}\right\rangle_{\kappa}=\kappa\left\langle D_{\perp}\right\rangle_{\kappa}$. The above formula can be then rewritten using the first cumulant (32) and eq. (A4) as

$$
(\star)=\left\langle\left|C-\Gamma_{1}\right|^{2}\right\rangle_{\kappa}=\left\langle\left(C-\Gamma_{1}\right)\left(C^{*}-\Gamma_{1}\right)\right\rangle_{\kappa}=\left\langle|C|^{2}\right\rangle_{\kappa}+\Gamma_{1}^{2}-2 \Gamma_{1}\langle\operatorname{Re}(C)\rangle_{\kappa}=\Gamma_{2},
$$

where we have used the fact that $\langle\operatorname{Re}(C)\rangle_{\kappa}=\Gamma_{1}$. The second term in the cumulant expansion is therefore nonnegative, as it should be. 


\section{Appendix B: Short time expansion of EACF with no hydrodynamic interactions}

In the 80s Lan, Ostrowsky and Sornette ${ }^{\frac{8}{}}$, in an early attempt to analyze the experimental data, developed an exact expression for $\hat{g}(\kappa, \boldsymbol{q} ; t)$ in case where the hydrodynamic interactions with the wall are neglected, i.e. $D$ is constant in the half-space occupied by the fluid and equals the single-particle bulk diffusion coefficient $D_{0}$. Their solution factorizes into terms corresponding to the decay in the direction parallel and perpendicular to the wall,

$$
\hat{g}(\boldsymbol{q}, \kappa ; t)=g_{\|}\left(q_{\|} ; t\right) g_{z}\left(q_{\perp}, \kappa ; t\right)
$$

The 'parallel' part is the usual expression for $\hat{g}$ in an unbounded fluid

$$
g_{\|}\left(q_{\|} ; t\right)=\exp \left\{-q_{\|}^{2} D_{0} t\right\}
$$

while the 'perpendicular' part can be conveniently written in terms of error function for complex argument $\underline{39} w(x)=e^{-x^{2}} \operatorname{erfc}(-i x)$ as

$$
g_{z}\left(q_{\perp}, \kappa ; t\right)=\frac{1}{2}\left[\left(1+\frac{i \kappa}{2 q_{\perp}}\right) w(i Z)+\left(1-\frac{i \kappa}{2 q_{\perp}}\right) w\left(i Z^{*}\right)\right],
$$

using a dimensionless quantity

$$
Z=\sqrt{D_{0} t}\left(i q_{\perp}+\frac{\kappa}{2}\right)=\frac{\kappa \sqrt{D_{0} t}}{2}\left(1+\frac{2 i q_{\perp}}{\kappa}\right)
$$

The variable $Z$ depends on two dimensionless parameters: $\kappa \sqrt{D_{0} t} / 2$ compares the electric field penetration depth with the distance diffused by the particle, and $2 q_{\perp} / \kappa$ compares the penetration depth with the length scale on which the system is probed. In case of no hydrodynamic interactions one can obtain an exact short-time expression of the EACF using the following representation of the error function for complex argument $\underline{39}$

$$
w(x)=\sum_{n=0}^{\infty} \frac{(i x)^{n}}{\Gamma(1+n / 2)},
$$

where $\Gamma$ denotes the Euler Gamma function. Subsequent terms of the expansion of $g_{\perp}$ are of the order $t^{n / 2}$, as $Z \sim \sqrt{t}$. Expanding both parts of $\hat{g}$ up to the order $t^{2}$, we get

$$
\begin{aligned}
g(\boldsymbol{q}, \kappa, t) & =1-D_{0}\left(\frac{\kappa^{2}}{4}+q_{\perp}^{2}+q_{\|}^{2}\right) t+\frac{4 D_{0}^{3 / 2}}{3 \sqrt{\pi}}\left(\frac{\kappa^{2}}{4}+q_{\perp}^{2}\right) t^{3 / 2} \\
& +\frac{D_{0}^{2}}{2}\left[q_{\|}^{4}+2 q_{\|}^{2}\left(\frac{\kappa^{2}}{4}+q_{\perp}^{2}\right)-\left(\frac{\kappa^{2}}{4}+q_{\perp}^{2}\right)\left(\frac{3 \kappa^{2}}{4}-q_{\perp}^{2}\right)\right] t^{2}+\mathrm{O}\left(t^{5 / 2}\right) .
\end{aligned}
$$


From this expansion, one identifies the first cumulant as

$$
\Gamma_{1}=D_{0}\left(q_{\|}^{2}+q_{\perp}^{2}+\frac{\kappa^{2}}{4}\right)
$$

which is in agreement with eq. (32) when $D_{\perp, \|}=D_{0}$. The next term contributes as $t^{3 / 2}$. Therefore, the second cumulant of $\hat{g}(\boldsymbol{q}, \kappa ; t)$ does not exist and cannot be obtained as a special case of eq. (36). The reason for this is the boundary condition on the wall, which is different in case where $D=D_{0}$ on the wall and $\mathbf{D}(z) \rightarrow \mathbf{0}$ as $z \rightarrow 0$ in presence of hydrodynamic interactions, as described in section III.

\section{REFERENCES}

${ }^{1}$ Z. Adamczyk Particles at Interfaces, Interactions, Deposition, Structure, Academic Press, 1998.

${ }^{2}$ K. C. Khilar, H. S. Fogler Migration of Fines in Porous Media, Kluwer Academic Publishers, 2006.

${ }^{3}$ T. M. Squires and S. R. Quake, Rev. Mod. Phys. 77, 977 (2005).

${ }^{4}$ D. Psaltis, S. R. Quake and C. Yang, Nature 442, 381 (2006).

${ }^{5}$ W. Norde and J. Lyklemma, Colloids and surfaces 38, 1 (1989).

${ }^{6}$ H. M. Lappin-Scatt, J. W. Costerton, ed., Microbial Biofilms, Cambridge University Press, 1995.

${ }^{7}$ I. H. Riedel, K. Kruse and J. Howard, Science 309, 300 (2005).

${ }^{8}$ K. H. Lan, N. Ostrowsky, and D. Sornette, Phys. Rev. Lett. 57, 17 (1986).

${ }^{9}$ R. Sigel, Curr. Opin. Coll. Interf. Sci. 14, 426 (2009).

${ }^{10}$ H. Brenner, Chem. Eng. Sci. 16, 242 (1961).

${ }^{11}$ A. J. Goldman, R. Cox, and H. Brenner, Chem. Eng. Sci. 22, 637 (1967).

${ }^{12}$ A. J. Goldman, R. Cox, and H. Brenner, Chem. Eng. Sci. 22, 653 (1967).

${ }^{13}$ P. Holmqvist, J. K. G. Dhont, and P. R. Lang, Phys. Rev. E 74, 021402 (2006).

${ }^{14}$ P. Holmqvist, J. K. G. Dhont, and P. R. Lang, J. Chem. Phys. 126, 044707 (2007).

${ }^{15}$ B. Cichocki, E. Wajnryb, J. Bławzdziewicz, J.K.G. Dhont and P.R. Lang, J. Chem. Phys. 132, 074704 (2010).

${ }^{16}$ J. W. Swan, J. F. Brady, J. Chem. Phys. 135, 014701 (2011).

${ }^{17}$ V. N. Michailidou, G. Petekidis, J. W. Swan and J. F. Brady, Phys. Rev. Lett. 102, 068302 (2009).

${ }^{18}$ H.A. Lorentz, Abhandlung über Theoretische Physics, Leipzig und Berlin, 1907. 
${ }^{19}$ H. Faxén, Ark. Mat. Astron. Fys. 17, 1 (1923).

${ }^{20}$ W. Dean, M. O’Neill, Mathematika 10, 13 (1963).

${ }^{21}$ M. O’Neill, Mathematika 11, 67 (1964).

${ }^{22}$ M. O’Neill, K. Stewartson, J. Fluid. Mech. 27, 705 (1967).

${ }^{23}$ B. Lin, J. Yu, i S. A. Rice, Phys. Rev. E 62, 10 (2000).

${ }^{24}$ R. Sadr, C. Hohenegger, H. Li, P. J. Mucha, and M. Yoda, J. Fluid Mech. 577, 443 (2007).

${ }^{25}$ P. Huang, K. Breuer, Phys. Rev. E 76, 046307 (2007).

${ }^{26}$ L. Lobry, N. Ostrowsky, Phys. Rev. B 53, 73808 (1996).

${ }^{27}$ K. Ishii, T. Iwai, and H. Xia, Optics Expr. 18, 7390 (2010).

${ }^{28}$ M. Plum, W. Steffen, G. Fytas, and W. Knoll, Optics Expr. 17, 10364 (2009).

${ }^{29}$ M. Plum, J. Rička, H. Butt, and W. Steffen, New J. Phys. 12, 103022 (2010).

${ }^{30}$ N. Ostrowsky and N. Garnier, Progr. Colloid Polym. Sci. 84, 371 (1991).

${ }^{31}$ N. Ostrowsky and L. Lobry, Il Nuovo Cimento D 16, 1111 (1994).

${ }^{32}$ M. Hosoda, K. Sakai, and K. Takagi, Phys. Rev. E 58, 6275 (1998).

${ }^{33}$ D. Frenkel, B. Smit, Understanding molecular simulations, Academic Press, 2002.

${ }^{34}$ B. Cichocki and R.B. Jones, Physica A 258, 273 (1998).

${ }^{35}$ G.S. Perkins, R.B. Jones, Physica A 171, 575 (1991).

${ }^{36}$ G.S. Perkins, R.B. Jones, Physica A 189, 447 (1992).

${ }^{37}$ S. Kim, S.J. Karrila, Microhydrodynamics: Principles and Selected Applications, Dover, 2005 .

${ }^{38}$ D.L. Ermak, J.A. McCammon, J. Chem. Phys. 69, 1352 (1978).

${ }^{39}$ M. Abramowitz, I. Stegun, ed., Handbook of Mathematical Functions, Dover, 1965.

${ }^{40}$ W. Steffen, MPI Mainz, Germany, personal communication. 